\title{
Things We Do For No Reason: Sliding-Scale Insulin as Monotherapy for Glycemic Control in Hospitalized Patients
}

\author{
Daniel B Ambrus, MD, MSc ${ }^{1,2 *}$; Mark J O'Connor, MD³
}

\begin{abstract}
${ }^{1}$ Department of Internal Medicine, Division of Hospital Medicine, UMass Memorial Medical Center, Worcester, Massachusetts; ${ }^{2}$ University of Massachusetts Medical School, Worcester, Massachusetts; ${ }^{3}$ Department of Internal Medicine, Division of Endocrinology, Massachusetts General Hospital, Boston, Massachusetts.
\end{abstract}

The "Things We Do for No Reason" (TWDFNR) series reviews practices that have become common parts of hospital care but may provide little value to our patients. Practices reviewed in the TWDFNR series do not represent "black and white" conclusions or clinical practice standards but are meant as a starting place for research and active discussions among hospitalists and patients. We invite you to be part of that discussion.

\section{A CLINICAL SCENARIO}

A 60-year-old man with a past medical history of obesity and type 2 diabetes presented to the emergency department with one week of myalgias and fever up to $103.5^{\circ} \mathrm{F}\left(39.7^{\circ} \mathrm{C}\right)$. Other vital signs were normal. He had no localizing symptoms, and physical examination was unrevealing, except for a small scab from a tick bite sustained two weeks prior to symptom onset. Before admission, he had been managing his diabetes with metformin 1,000 mg twice a day, and on arrival, his blood sugar level was $275 \mathrm{mg} / \mathrm{dL}$. The admitting provider decided to hold the patient's metformin and replace it with insulin per a sliding scale. Is monotherapy with sliding-scale insulin the best inpatient management option for this patient's type 2 diabetes?

\section{WHY YOU MIGHT THINK SLIDING-SCALE INSULIN AS MONOTHERAPY IS HELPFUL}

The basic premise of sliding-scale insulin (SSI) is to correct hyperglycemia through the frequent administration of short-acting insulin dosed according to a patient's blood glucose level with the help of a prespecified rubric. When blood glucose levels are low, patients receive little or no insulin, and when blood glucose levels are high, higher doses are given. This approach to inpatient blood glucose management was first popularized by Joslin in 1934, ${ }^{1}$ and it remains a common strategy today. For example, a 2007 survey of 44 hospitals in the United States showed that approximately $43 \%$ of all noncritically ill patients with hyperglycemia were treated with SSI alone. ${ }^{2}$ More recently, a single-center study showed that $30 \%$ of clinicians continued to use SSI as monotherapy even after the implementation

*Corresponding Author: Daniel B. Ambrus, MD, MSc; E-mail: daniel.ambrus@ umassmemorial.org; Telephone: 508-334-8515; Twitter: @ambrus07

Published online first November 28, 2018

Received: June 15, 2018; Revised: October 1, 2018;

Accepted: October 12, 2018

() 2019 Society of Hospital Medicine DOI 10.12788/jhm.3109 of order sets designed to limit this practice. ${ }^{3}$

The rationale for SSI as monotherapy appears to have two components. First, guidelines suggest that certain patients should be screened periodically in the hospital for hyperglycemia (blood glucose persistently greater than $180 \mathrm{mg} / \mathrm{dL}$ ) and that, if identified, hyperglycemia should be treated. ${ }^{4}$ By pairing finger-stick glucose monitoring with $\mathrm{SSI}$, the diagnosis and treatment-although not the prevention-of hyperglycemia can be accomplished simultaneously. Second, inpatient providers do not want to cause harm in the form of hypoglycemia. SSI as monotherapy is sometimes viewed as a cautious approach in this regard as insulin is administered only if the blood sugar level is high.

Convenience is probably another key contributor to the enduring use of SSI as monotherapy. Several hospitals have ready-made order sets for SSI that are easier to prescribe than a patient-specific regimen including both short- and long-acting insulin. In at least one single-center survey, physicians and staff were found to favor convenience over perceived efficacy when asked about their attitudes toward inpatient glycemic control..$^{5}$ Although efforts at individual hospitals to change practice patterns among residents have shown promise, ${ }^{6}$ reform on a broader scale remains elusive.

\section{WHY SSI AS MONOTHERAPY IS NOT HELPFUL}

SSI administration does not attempt to replicate normal pancreatic physiology, which involves basal insulin secretion to impair hepatic gluconeogenesis and meal-associated insulin spikes to promote uptake into glucose-avid tissues. SSI is a reactive strategy, not a proactive one, and perhaps unsurprisingly, to our knowledge, it has never been shown to prevent hyperglycemia in hospitalized patients, an impression corroborated by a systematic review of the topic between 1964 and 2003. ${ }^{7}$ More recently, one multicenter trial analyzed the effect of adding SSI to oral antihyperglycemic medications in hospitalized diabetics and found no differences in rates of hyperglycemia. ${ }^{8}$ Another study found that $84 \%$ of administered SSI doses failed to correct hyperglycemia. ${ }^{9}$

However, does adding basal insulin to SSI raise a patient's risk of hypoglycemia? When basal insulin is dosed carefully, the answer appears to be no. In a trial in which diabetic long-term care residents who were receiving SSI at baseline were randomized to either continued SSI or basal-bolus insulin, the investigators found that the basal-bolus group experienced significantly lower average blood glucose levels without an increase in adverse 
glycemic events. ${ }^{10}$ Perhaps the most significant milestone to date, however, was the RABBIT 2 multicenter trial, published in 2007, that randomized hospitalized, insulin-naive diabetics to either a weight-based regimen of basal and prandial insulin or SSI only. ${ }^{11}$ Rates of hypoglycemia and length of stay did not differ between the groups, and $66 \%$ of patients receiving basal-prandial insulin achieved their glycemic control target as opposed to just $38 \%$ of patients in the SSI-only group. The SSI group also required more total insulin. A weight-based, basal-bolus strategy was later proven to be similarly effective, without causing severe hypoglycemia, for patients undergoing surgery who could not maintain consistent oral alimentation. ${ }^{12}$ Basal-bolus insulin was associated with fewer surgical complications, and it produced a cost savings of $\$ 751$ per day as determined by a post hoc comparative effectiveness study. ${ }^{13}$

Prolonged use of SSI as monotherapy may be not only ineffective but also harmful. Clearly, the absence of basal insulin will harm type 1 diabetics, who need basal insulin to prevent diabetic ketoacidosis. However, even for type 2 diabetics and nondiabetics, hyperglycemia has been established as a marker for adverse outcomes among hospitalized patients, ${ }^{14}$ and SSI monotherapy has been associated with a three-fold higher risk of hyperglycemia compared with the use of a sliding scale plus other forms of insulin. ${ }^{15}$ At least one other study has also linked this practice with a significantly increased length of stay compared with patients who were receiving insulin proactively. ${ }^{16}$ We believe that the potential for harm is difficult to disregard, especially because safer alternatives are available. Ultimately, it can be stated that in hospitalized patients with persistent hyperglycemia who require insulin, SSI alone should not be the preferred treatment choice regardless of whether the patient carries a known diagnosis of diabetes mellitus or has used insulin previously.

\section{WHEN YOU MIGHT CONSIDER USING SSI AS MONOTHERAPY}

As discussed above, there is no known clinical scenario in which SSI as monotherapy has been proven to be effective; however, the use of SSI as monotherapy as a short-term approach has not been well studied. Hospitalized patients who are at risk for adverse glycemic events should be monitored with periodic finger-stick blood glucose draws per guidelines. In the first 24 hours, it may be reasonable to withhold basal insulin for insulin-naive patients, particularly if the medication reconciliation or other key components of the history are in doubt or if there are risk factors for hypoglycemia such as a history of bariatric surgery. The amount of insulin received in the first 24 hours of such monitoring may inform subsequent insulin dosing, but this method of "dose finding" has not been validated in the literature.

Uncertain or interrupted alimentation status or stress hyperglycemia may complicate the assessment of a patient's insulin needs. One of the insights from the RABBIT 2 surgery trial is that even with interrupted alimentation, patients on a weightbased, long-acting insulin regimen did not experience severe hypoglycemia. Nevertheless, if a patient without type 1 diabetes is felt to be at high risk for a severe hypoglycemic event, it may be prudent to withhold long-acting insulin. However, in that situation, adding SSI to finger-stick monitoring is unlikely to be beneficial. Cases of stress hyperglycemia in nondiabetics can also be challenging, as the persistence of hyperglycemia can be difficult to predict. Guidelines state that if hyperglycemia is persistent, then insulin therapy should be initiated and that this therapy is best accomplished in the form of a basal-prandial regimen. ${ }^{17}$

\section{WHAT YOU SHOULD DO INSTEAD}

Current guidelines from the American Diabetes Association ${ }^{17}$ and the American Association of Clinical Endocrinologists ${ }^{18}$ for hospitalized patients with hyperglycemia who require insulin recommend against the prolonged use of SSI as monotherapy (category A recommendation) and support the use of basal plus correctional insulin with the addition of nutritional insulin for patients with consistent oral intake (category A recommendation). Although a complete discourse on the determination of the appropriate starting dose of insulin is outside of the scope of this case presentation, the basic approach begins with calculating a weight-based total daily dose of insulin, approximately half of which can be given as basal insulin with the remainder given with meals along with correctional insulin as needed to account for premeal hyperglycemia. ${ }^{4}$ For example, the protocol used in the RABBIT 2 trial, which involved known type 2 diabetics, started insulin based on a total daily dose of 0.4 units/ $\mathrm{kg}$ for patients presenting with blood sugar levels $\leq 200 \mathrm{mg} / \mathrm{dL}$ and 0.5 units $/ \mathrm{kg}$ for those with higher initial glucose levels. ${ }^{7}$ Half of the total daily dose was given as basal insulin, and the other half was divided among meals. Caution with insulin dosing may be required in patients aged $>70$ years, in those with impaired renal function, and in situations in which steroid doses are fluctuating. The Society of Hospital Medicine has formulated an online subcutaneous insulin order implementation guideline, eQUIPS, that can be a helpful resource to centers that are interested in changing their practice patterns. ${ }^{19}$

\section{RECOMMENDATIONS}

- Instead of using SSI monotherapy for hospitalized patients who require insulin, add basal and prandial insulin, using a weight-based approach if necessary for insulin-naive patients.

- Engage with leadership at your center to learn how inpatient hyperglycemia protocols and blood sugar management teams can help provide evidence-based and individualized treatment plans for your patients.

- If no infrastructure exists at your center, the Society of Hospital Medicine offers training and guidance through its eQUIPS inpatient hyperglycemia management program.

\section{CONCLUSION}

In the case presentation, the hyperglycemic patient whose metformin was on hold should have been started on a combination of basal and prandial insulin as determined by his weight and current renal function as opposed to monotherapy with SSI. Using SSI as monotherapy for hyperglycemia is a 
common practice, and although well-intentioned, it is an ineffective and possibly dangerous approach. Continued efforts must be made to address the gap between guidelines and suboptimal practice patterns locally and nationally.

Do you think this is a low-value practice? Is this truly a "Thing We Do for No Reason?" Share what you do in your practice and join in the conversation online by retweeting it on Twitter (\#TWDFNR) and liking it on Facebook. We invite you to propose ideas for other "Things We Do for No Reason" topics by emailing TWDFNR@hospitalmedicine.org.

\section{Acknowledgments}

The authors would like to thank Dr. Asem Ali of the Division of Endocrinology at UMass Memorial Medical Center for his review of the manuscript.

Disclosures: The authors have nothing to disclose.

\section{References}

1. Joslin EP. A Diabetic Manual for the Mutual Use of Doctor and Patient. Philadelphia, PA: Lea \& Febiger; 1934:108.

2. Wexler DJ, Meigs JB, Cagliero E, Nathan DM, Grant RW. Prevalence of hyper- and hypoglycemia among inpatients with diabetes: a national survey of 44 U.S. hospitals. Diabetes Care. 2007;30(2):367-369. doi: 10.2337/dc06-1715.

3. Valgardson JD, Merino M, Redgrave J, Hudson JI, Hudson MS. Effectiveness of inpatient insulin order sets using human insulins in noncritically ill patients in a rural hospital. Endocr Pract. 2015;21(7):794-806. doi: 10.4158/EP14153.

4. Clement S, Braithwaite SS, Magee MF, et al. Management of diabetes and hyperglycemia in hospitals. Diabetes Care. 2004;27(2):553-591. doi: 10.2337/ diacare.27.2.553.

5. Beliard R, Muzykovsky K, Vincent W, 3rd, Shah B, Davanos E. Perceptions, barriers, and knowledge of inpatient glycemic control: a survey of health care workers. J Pharm Pract. 2016;29(4):348-354. doi: 10.1177/0897190014566309.

6. Baldwin D, Villanueva G, McNutt R, Bhatnagar S. Eliminating inpatient sliding-scale insulin: a reeducation project with medical house staff. Diabetes Care. 2005;28(5):1008-1011. doi: 10.2337/diacare.28.12.2987-a.

7. Browning LA, Dumo P. Sliding-scale insulin: an antiquated approach to glycemic control in hospitalized patients. Am J Health Syst Pharm. 2004;61(15):1611-1614.
8. Dickerson LM, Ye X, Sack JL, Hueston WJ. Glycemic control in medical inpatients with type 2 diabetes mellitus receiving sliding scale insulin regimens versus routine diabetes medications: a multicenter randomized controlled trial. Ann Fam Med. 2003;1(1):29-35

9. Golightly LK, Jones MA, Hamamura DH, Stolpman NM, McDermott MT. Management of diabetes mellitus in hospitalized patients: efficiency and effectiveness of sliding-scale insulin therapy. Pharmacotherapy. 2006;26(10):14211432. doi: 10.1592/phco.26.10.1421.

10. Dharmarajan TS, Mahajan D, Zambrano A, et al. Sliding scale insulin vs basal-bolus insulin therapy in long-term care: a 21-day randomized controlled trial comparing efficacy, safety and feasibility. J Am Med Dir Assoc. 2016;17(3):206-213. doi: 10.1016/j.jamda.2015.08.015.

11. Umpierrez GE, Smiley D, Zisman A, et al. Randomized study of basal-bolus insulin therapy in the inpatient management of patients with type 2 diabetes (RABBIT 2 trial). Diabetes Care. 2007;30(9):2181-2186. doi: 10.2337/dc070295.

12. Umpierrez GE, Smiley $D$, Jacobs $S$, et al. Randomized study of basal-bolus insulin therapy in the inpatient management of patients with type 2 diabetes undergoing general surgery (RABBIT 2 surgery). Diabetes Care. 2011;34(2):256-261. doi: 10.2337/dc10-1407.

13. Phillips VL, Byrd AL, Adeel S, Peng L, Smiley DD, Umpierrez GE. A comparison of inpatient cost per day in general surgery patients with type 2 diabetes treated with basal-bolus versus sliding scale insulin regimens. Pharmacoecon Open. 2017;1(2):109-115. doi: 10.1007/s41669-017-0020-9..

14. Umpierrez GE, Isaacs SD, Bazargan N, You X, Thaler LM, Kitabchi AE. Hyperglycemia: an independent marker of in-hospital mortality in patients with undiagnosed diabetes. J Clin Endocrinol Metab. 2002;87(3):978-982. doi: 10.1210/jcem.87.3.8341.

15. Queale WS, Seidler AJ, Brancati FL. Glycemic control and sliding scale insulin use in medical inpatients with diabetes mellitus. Arch Intern Med. 1997; 157(5):545-552.

16. Gearhart JG, Duncan JL, 3rd, Replogle WH, Forbes RC, Walley EJ. Efficacy of sliding-scale insulin therapy: a comparison with prospective regimens. Fam Pract Res J. 1994;14(4):313-322.

17. American Diabetes A. 14. Diabetes care in the hospital: Standards of medical care in diabetes-2018. Diabetes Care. 2018;41(Suppl 1):S144-S151. doi: 10.2337/dc18-S014.

18. Moghissi ES, Korytkowski MT, DiNardo M, et al. American Association of Clinical Endocrinologists and American Diabetes Association consensus statement on inpatient glycemic control. Endocr Pract. 2009;15(4):353-369. doi: $10.2337 / \mathrm{dc} 09-9029$.

19. Maynard G, Wesorick DH, O'Malley C, Inzucchi SE, Society of Hospital Medicine Glycemic Control Task F. Subcutaneous insulin order sets and protocols: effective design and implementation strategies. J Hosp Med. 2008;3(5 Suppl):29-41. doi: 10.1002/jhm.354. 